\title{
Tangential forces in the contact area of upper road layer with the base
}

\author{
Timmo Gavrilov ${ }^{1, *}$, Gennady Kolesnikov ${ }^{2}$ and Konstantin Khoroshilov ${ }^{2}$ \\ ${ }^{1}$ Emperor Alexander Ist. Petersburg State Transport University, 190031, Saint Petersburg, Moskovsky \\ pr., 9, Russia \\ ${ }^{2}$ Petrozavodsk State University, 185910, Petrozavodsk, Lenin pr., 33, Russia
}

\begin{abstract}
Low-temperature cracking is one of the main reasons for the deterioration of the upper layer of roads in regions with cold winters. A large number of works focused on deterioration prevention are known, however the interdisciplinary problem of low-temperature cracking remains relevant. The important, but insufficiently studied factor is the distribution of normal and tangential (shear) forces acting on the upper layer of the road. The objective of the current study was to clarify insight about the distribution of tangential forces in the contact area of the upper layer of the road with the base. There are works in which it is assumed that these forces are distributed by a piecewise linear law. We propose a mechanical and mathematical model and an analytical solution to the problem of the distribution of shear forces in the contact area of the upper layer with the base. The main result of the study: it is found that the distribution of shear forces in the segment of the asphalt concrete layer is described by a second-order polynomial. However, the shear stresses are distributed linearly over the entire length of the segment and reach the highest modulo values in the area of the end sections of the segment. The results of the presented study clarify the understanding of functioning of the upper road layer at low temperatures. By that, the contribution to the solution of the problem of increasing the crack resistance of roads in regions with cold winters is made.
\end{abstract}

\section{Introduction}

Low-temperature cracking is one of the main reasons for the deterioration of the roads upper layer in regions with cold winters. A large number of works focused on deterioration prevention are known, however the interdisciplinary problem of low-temperature cracking remains relevant. Low-temperature cracks, also called frost cracks, are formed in the upper layers of soils during their freezing [1], in asphalt concrete pavement of the road [2] and airfields [3]. Studies [2, 3] showed that the important, but insufficiently studied factor is the distribution of normal [5] and tangential (shear) forces [6] acting on the upper layer of the road. Monitoring the condition of the roads is performed with the use of physical measurements and associated devices [2]. The results of measurements are also necessary

\footnotetext{
*Corresponding author: gtimmo@mail.ru
} 
to check the adequacy of mathematical models $[7,8]$, which are developed to predict the stresses and strains of road surfaces $[3,4,9]$.

The current study discusses the low-temperature cracks of asphalt concrete road surfaces. The peculiarity of such cracks is that they appear a few years after the completion of the road and are oriented perpendicular to the longitudinal axis of the road. This feature allows using one-dimensional models of a rectangular segment of the road surface in theoretical studies, i.e. to consider this segment as a straight rod that is opposed to temperature and force effects $[8,10]$. The length of the segment, and hence the length of the specified rod, is determined by the distance between the cracks.

The distance between the cracks varies from several meters to tens of meters, depending on the physical and mechanical properties of asphalt concrete [11], on the amplitude and speed of temperature changes [2], as well as on the thickness of the asphalt layer and other parameters $[3,4]$.

When the upper layer of the road is frozen, the length of the segment decreases, but the tangential forces of friction and adhesion in the contact area of the segment with the base resist thermal deformations, but do not eliminate them. With increasing tangential forces, the tensile forces increase in the upper layer material, which can cause its destruction and the appearance of a crack [2-8]. Thus, the tangential forces of friction and adhesion in the contact area of the base and the upper layer significantly affect its state.

However, in the known works the relevant aspects are not fully investigated. For example, there are works in which it is assumed that the tangent forces in the contact area of the upper layer of the road with the base are distributed by a piecewise linear law [10]. In this regard, taking into account the results of [6], it is necessary to study the restrictions on the volume of the longitudinal linear law in the simulation of the distribution of the tangential forces acting in the area of contact of the upper layer of the road with the base. Accordingly, it is legitimate to formulate a assumption of current study: the tangential forces acting in the area of contact of the upper layer of the road with the base are not always distributed according to the piecewise linear law.

The objective of the current study was to clarify insight about the distribution of tangential forces in the contact area of the upper layer of the road with the base.

\section{Materials and Methods}

\subsection{Tangential forces in the contact area of upper layer with the base}

You are free to use colour illustrations for the online version of the proceedings but any print version will be printed in black and white unless special arrangements have been made with the conference organiser. Please check whether or not this is the case. If the print version will be black and white only, you should check your figure captions carefully and remove any reference to colour in the illustration and text. In addition, some colour figures will degrade or suffer loss of information when converted to black and white, and this should be taken into account when preparing them.

By analogy with [8], the segment of the asphalt concrete layer is modeled by an elastic homogeneous rod of width $B$, thickness $H$ and length $L=2 l$ (Fig. 1). For the rod material, the modulus of elasticity $E$, the Poisson's ratio $v$ and the linear coefficient of thermal contraction/expansion $\alpha$ coefficient of linear thermal expansion $\alpha$ are known. Specific values of asphalt concrete characteristics depending on the temperature can be found, for example, in works $[5,7,8,11,12]$. It is assumed that the temperature is the same for all points on the day surface of the asphalt concrete layer. It is also assumed that the value of $H$ is small enough to ignore the unevenness of the temperature distribution over the thickness 
of the layer. When freezing, the length of the segment is reduced by $2 \Delta l$. However, the deformation of the asphalt concrete segment is limits by friction forces and adhesion of $T$ with the underlying layer. In this model, the lines of action of these forces are located in the plane of contact of the layers. These forces are proportional to the stiffness $k$ of the elastic base layer. The stiffness $k$ depends on a number of factors, can be determined experimentally or from literature. For example, for one of the variants of concrete covering $k=416 \mathrm{MH} / \mathrm{m}^{3}$ [8]. The stiffness $k$ characterizes the resistance to displacement only in the horizontal direction. From the physical point of view, the value of $k$ can be considered as the stiffness $K$ of a usual spring (dimension $\mathrm{N} / \mathrm{m}$ ), but distributed over the area $a$ of the contact layers: $k=K / a$. Hence for the element with length $d x$ and width $B$ (Fig. 1) we can write: $a=B d x$,

$$
K=k B d x
$$

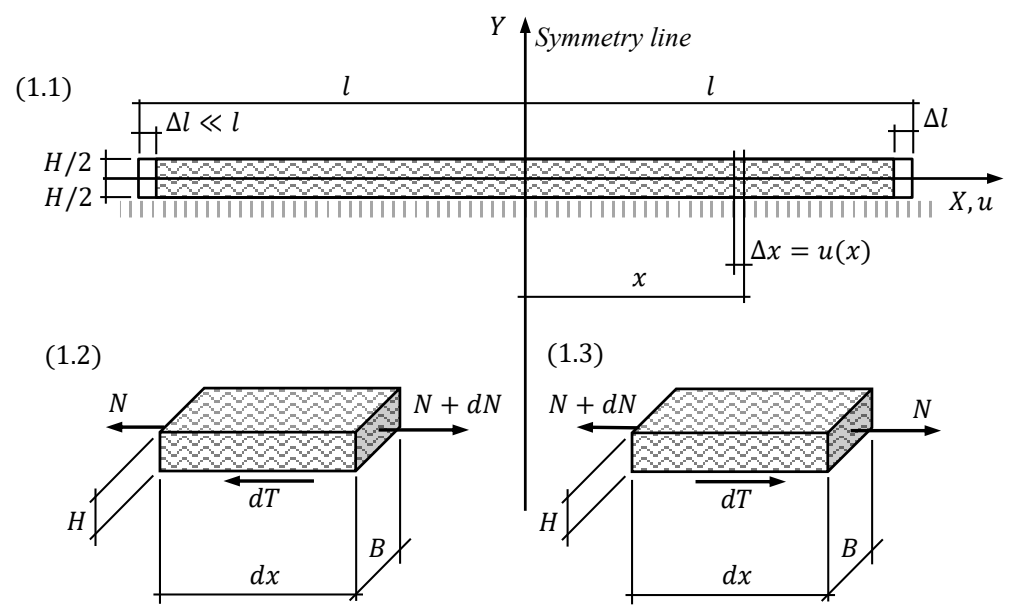

Fig. 1. The scheme of the upper layer (1.1) and its symmetric elements (1.2 and 1.3).

The change in the segment temperature by $\Delta T$ degrees is associated with the movement of a point $i$ relationship $u=\Delta x$, где $\Delta x=\alpha x \Delta t$.

The change $d u$ of the longitudinal deformation of the element of length $d x$ (Fig. 1), for which the cross-sectional area is $A=B H$, and the modulus of elasticity of the material is $E$, due to two factors, namely the change in temperature $\Delta \mathrm{t}$ and the increment $d N$ of the longitudinal force $N$. Thus, $d u=\alpha \Delta t d x+d N d x /(E A)$. Taking into account only the first order smallness values, we write $d u \approx \alpha \Delta t d x$. Integrating and taking into account that $u=0$ at $x=0$ (Fig.1), we obtain:

$$
u=\alpha \times \Delta t
$$

Due to the symmetry of the physical and geometric parameters of the model (Fig. 1), the internal forces and displacements are mirror-symmetrical with respect to the $Y$ axis: $u(-x)=-u(x)$.

From the equilibrium condition of an element with length $d x$ it follows that it is up to a sign

$$
d T=d N
$$

The magnitude of the tangent force $d T$, acting on the area of contact of the element under consideration with the base, is proportional to its length $d x$, width $B$, stiffness of the 
base $k$ in the horizontal direction and the movement $u$ in the same direction. Thus, we can write: $d T=K u$, where $K$ and $\mathrm{u}$ are defined by (1) and (2), respectively: $d T=(k B d x) u$. Then, taking into account (2), we obtain:

$$
d T=k B \alpha \Delta t x d x .
$$

We determine the intensity of the shear forces $T_{i n t}=d T / d x$. Given (4), write down:

$$
T_{\text {int }}=k B \alpha \Delta t x .
$$

It follows from (5) that the intensity of shear forces reaches the highest modulo values at $x= \pm l$ (Fig. 1). If $x=0$, then $T_{\text {int }}$, and hence the shear force $T$ is zero.

Integrating both parts of equality (4), we obtain: $T=k B \alpha \Delta t x^{2} / 2+C_{1}$. The integration constant $C_{1}$ can be found, taking into account (5): if $x=0$, then $T_{\text {int }}=0$, and hence the shear force $T$ is zero. Thus, $C_{1}=0$,

$$
T=k B \alpha \Delta t x^{2} / 2 .
$$

Since at the freezing $\Delta t<0$, from (6), it follows that the tangential forces $T$ in the contact area of the upper layer with the base reach the highest modulo values, if $x= \pm l$. In the segment length middle, the force $T=0$ (if $x=0$ by Fig. 1).

We find the shear stresses $\tau=d T / d A$. Here $d A=B d x$. Taking into account (4) we obtain:

$$
\tau=k \alpha \Delta t x
$$

If $x=0$, then, according to (7), the tangential stresses are zero at any value of $\Delta t$. If a fixed value of $\Delta t \neq 0$, the tangential stresses increase linearly in modulus $x$ and reach the highest values at $x= \pm l$.

\subsection{The relationship of tangential and tensile forces}

The shear force $T$ associated with the normal force $N$ (Fig. 1) differential ratio (3). Using (3) and (4), we write:

$$
d N=k B \alpha \Delta t x d x
$$

Integrating both parts of equality (8) we obtain: $N=k B \alpha \Delta t x^{2} / 2+C_{2}$. In the physical model of the segment (Fig. 1) there are no longitudinal forces on the end faces of the segment: if $x= \pm l$, then $N=0$. Thus, $C_{2}=-k B \alpha \Delta t l^{2} / 2$.

$$
N=k B \alpha \Delta t\left(x^{2}-l^{2}\right) / 2 .
$$

Here $x^{2} \leq l^{2}$; if $\Delta t<0, N \geq 0$. The "+" sign indicates that the direction of the force $N$ coincides with the direction indicated in Fig. 1, i.e. the force $N$ causes a tensile of the segment. Extremum $N=N(x=0)=-k B \alpha \Delta t l^{2} / 2$.

Using (9), we find the normal stresses $\sigma$ in the cross section of the segment in Fig. 1. $\sigma=N / A$, where $A=B H$ :

$$
\sigma=k \alpha \Delta t\left(x^{2}-l^{2}\right) /(2 H) \text {. }
$$

\section{Results}

\subsection{Numerical analysis of tangential and tensile forces and stresses}


Consider an example of using formulas (6), (7), (9) and (10). Realistic data have been taken from literature. Let $L=12 \mathrm{~m}, B=3.6 \mathrm{~m}, H=0.09 \mathrm{~m}, \alpha=2.5 \cdot 10^{-5}\left(1 /{ }^{\circ} \mathrm{C}\right.$ ) [10, p. 749], $\Delta t=-10^{\circ} \mathrm{C}$. We consider two possible options: $k=35 \mathrm{MPa}$ and $k=15 \mathrm{MPa}$. The results of the calculations are shown in Fig. $2-5$.

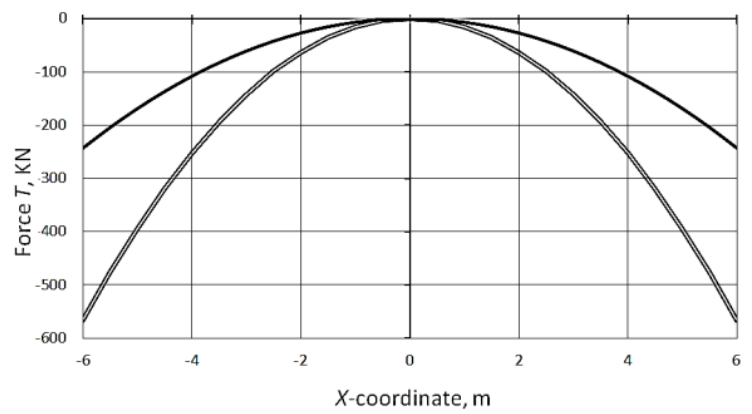

$-\mathrm{T}$, if $\mathrm{k}=15 \mathrm{Mpa} \quad \mathrm{T}$, if $\mathrm{k}=35 \mathrm{Mpa}$

Fig. 2. Tangential force $T$ for a segment length of $12 \mathrm{~m}$ by Fig. 1.

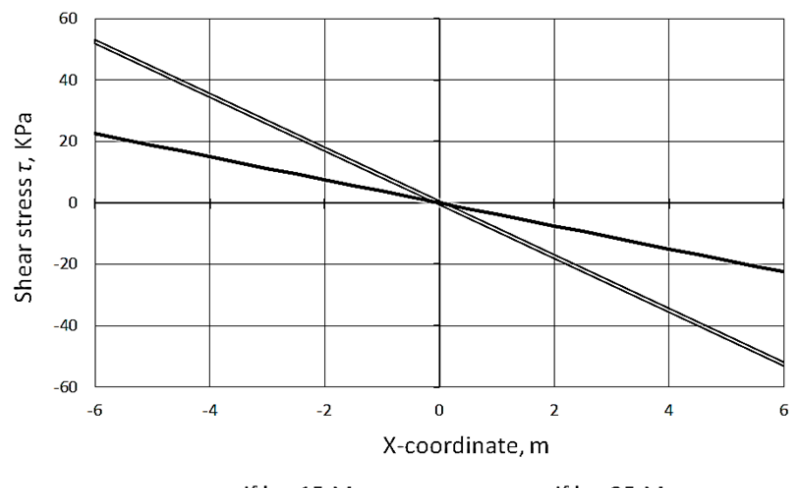

- $\tau$, if $k=15 \mathrm{Mpa} \quad \tau$, , if $k=35 \mathrm{Mpa}$

Fig. 3. Tangential stress $\tau$ for a segment length of $12 \mathrm{~m}$ by Fig. 1 .

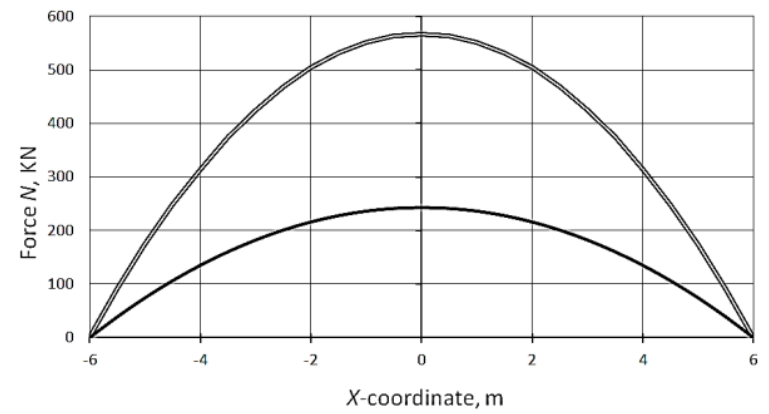

$-\mathrm{N}$, if $\mathrm{k}=15 \mathrm{Mpa} \quad \mathrm{N}$, if $\mathrm{k}=35 \mathrm{Mpa}$

Fig. 4. Tensile forces $N$ for a segment length of $12 \mathrm{~m}$ by Fig. 1 . 


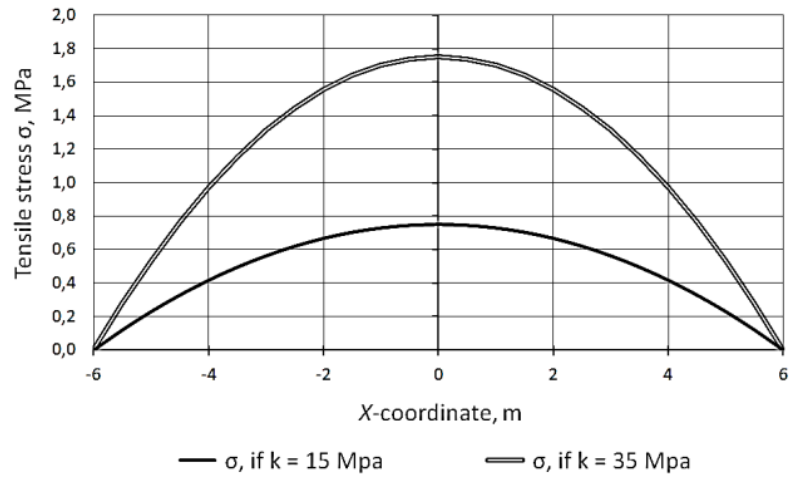

Fig. 5. Tensile stress 6 for a segment length of $12 \mathrm{~m}$ by Fig. 1 .

\subsection{Tangential forces and slippage}

It is known that when the temperature decreases, the shear stress in the contact zone of the upper layer with the base (7) will increase linearly modulo. However, the growth is limited to a certain value $\tau 0$ (Fig. (6)). The appropriate aspects are described in more detail in article [6]. Given this fact, it should be noted that the relations (6) and (7) are obtained under the condition $\tau<\tau_{0}$.

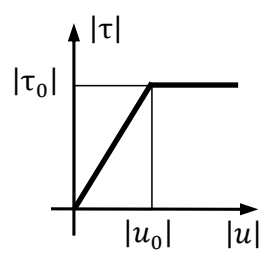

Fig. 6. Limited increase of tangential stress.

Consider the case when $|\tau|=\left|\tau_{0}\right|$. Fig. 6 shows that if in some area of contact $|\tau|=\left|\tau_{0}\right|$, from a physical point of view in this area is slippage of the top layer. The value of $\tau_{0}$ is limited by the finite values of the friction force and the adhesion force [13]. Figures 2 and 3 show that the tangent stresses are small in the middle of the segment and therefore there is no slippage. However, near the ends of the segment, sliding is possible because the shear forces and their corresponding shear stresses reach the highest values.

Let us denote $x_{0}-$ coordinate of the point where $|\tau|=\left|\tau_{0}\right|$. We find $x_{0}$.

If $x=x_{0}$, use (6) to write:

$$
T=k B \alpha \Delta t x_{0}^{2} / 2
$$

On the other hand, if $\tau=\tau_{0}$, then $d T_{0}=\tau_{0} d A$, where $d A=B d x$. Integrating, we write:

$$
T_{0}=\tau_{0} B x+C_{2}
$$

From the physical point of view, derivatives are continuous in the problem under consideration for $x=x_{0}$. Therefore, using (11) and (12), can be written:

$$
\begin{gathered}
d T / d x=d T_{0} / d x, x=x_{0} . \\
k B \alpha \Delta t x_{0}=\tau_{0} B .
\end{gathered}
$$




$$
x_{0}=T_{0} /(k \alpha \Delta t) .
$$

Then, taking into account the symmetry (Fig. 1), we obtain:

$$
x_{0}= \pm T_{0} /(k \alpha \Delta t) .
$$

Thus, if $0 \leq x \leq x_{0}$ or $x_{0} \leq x \leq l$, implement the slippage, i.e. the displacement $u$ grow at constant $|\tau|=\left|\tau_{0}\right|$ (Fig. 6).

The value of $k$ can be found by the formula

$$
k=\tau_{0} / u_{0}
$$

The values $\tau_{0}$ and $u_{0}$ are determined experimentally or from literature. So, for asphalt concrete AC-S: $\tau_{0}=0.021 \mathrm{MPa}, u_{0}=0.60 \mathrm{~mm}$; for asphalt concrete AS: $\tau_{0}=0.015 \mathrm{MPa}$, $u_{0}=1.00 \mathrm{~mm}$ [6, p. 458]. Accordingly, by the formula (17) can be find two values $k$ : $35 \mathrm{MPa}$ and $15 \mathrm{MPa}$. These values were used in the above example (Fig. 2-5). For this example, using (17), we obtain $x_{0}= \pm 4.0 \mathrm{~m}$ in the first case and $x_{0}= \pm 2.4 \mathrm{~m}$ in the second case. Thus, the reduction of adhesion of the upper layer of the road structure with the base layer leads to a decrease of the shear zone.

\section{Discussion}

The segment of the upper layer of the road is considered when it is frozen. A simple mechanical and mathematical model and an analytical solution to the problem of distribution of shear forces in the contact zone of the segment of road with the base are proposed. Two cases are investigated, in one of which the tangent stresses are less than the limit values. In the other case, the tangential (shear) stress is equal to the limit value.

It is found that in the first case the distribution of shear forces in the segment of the asphalt concrete layer is described by a polynomial of the second order. However, the shear stresses are distributed linearly over the entire length of the segment and reach the highest modulo values in the region of the end sections of the segment.

In the second case, the shear forces increase linearly from the middle of the segment length to its ends, and the shear stress remains constant. Such a condition is possible on the part of the length of the segment.

The coordinates of the point at which the tangential stress reaches the limit value are determined.

The presented studies have shown that the reduction of stiffness ( $k$ in the formulas (1), (4) - (16)), that is, the reduction of friction and adhesion of the upper layer of road structure in its interaction with the base leads to a decrease in shear and tensile forces (Fig. 2 - 5). This partially eliminates the causes of new cracks in the freezing of the upper layer of the road structure. A similar result, but for reflected cracks, is justified in [3, p. 72]. Thus, the application of the results of the study extends the possibility of increasing the crack resistance of road structure upper layer when they seasonal freezing.

\section{Conclusions}

The analytical solution of the problem of the distribution of tangent forces in the zone of contact of the upper road layer with the base is considered. It was shoved that the distribution of tangential forces in the contact area of the segment of the asphalt concrete layer with the base is described by a polynomial of the second order, if the shear stresses do not exceed the pedal values. 
The obtained relations for determining the value forces and stresses acting in the zone of contact of the upper layer of the road structure with the underlying layer can be used in determining the parameters of the layers road structure at the design stage.

The prospects of this study are associated with the analysis of the effect of friction and adhesion, taking into account their dependence on the temperature when freezing the upper layer of the road $[14,15]$.

\section{References}

1. V.P. Merzlyakov, Soil Mechanics and Foundation Engineering, 53, 221-225 (2016). DOI: $10.1007 / \mathrm{s} 11204-016-9389-1$

2. B. Teltayev, Reports of the national Academy of sciences of the Republic of Kazakhstan, 5, 35-57 (2015)

3. A.N. Popov, A.V. Kochetkov, A.N. Masalykin, Russian Journal of Building Construction and Architecture, 2, 65-74 (2015)

4. P. Pereira, J.A. Pais, Journal of Traffic and Transportation Engineering, 4, 316-346 (2017) https://doi.org/10.1016/j.jtte.2017.06.001

5. D. Rys, J. Judycki, M. Pszczola, M. Jaczewski, L. Mejlun, Construction and Building Materials, 147, 478-487 (2017) https://doi.org/10.1016/j.conbuildmat.2017.04.179

6. J. Zhang, V.C. Li, Journal of Transportation Engineering, 127, 455-462 (2001)

7. M. Pszczoła, J. Judycki, Baltic Journal of Road \& Bridge Engineering, 10, 39-45 (2015) DOI: 10.3846/bjrbe.2015.05

8. G. Chen, G. Baker, Advances in Structural Engineering, 8, 595-602 (2005) http://dx.doi.org/10.1260/136943305776318338

9. M.A. Zavyalov, A.M. Kirillov, Magazine of Civil Engineering, 54, 70-76 (2015). DOI: 10.18720/MCE.70.5

10. P. Rajbongsh, A. Das, Journal of Transportation Engineering, 135, 745-752 (2009)

11. G.M. Levashov, V.V. Sirotuk, Vestnik SibADI, 22, 23-26 (2011)

12. J. Judycki, International Journal of Pavement Engineering, 19, 24-36 (2018) https://doi.org/10.1080/10298436.2016.1149840

13. N.K. Myshkin, I.G. Goryacheva, Journal of Friction and Wear, 37, 513-516 (2016) DOI: $10.3103 / \mathrm{S} 106836661606009 \mathrm{X}$

14. G.N. Kirukhin, Roads and Bridges (Dorogi i mosty), 30, 309-328 (2013)

15. I.I. Dmitriev, A.M. Kirillov, Construction of Unique Buildings and Structures, 53, 728 (2017) 